\title{
Observed Area Under the Curve Percent
} Extrapolation

National Cancer Institute

\section{Source}

National Cancer Institute. Observed Area Under the Curve Percent Extrapolation. NCI

Thesaurus. Code C85764.

The percentage of the area under the curve (AUC) extrapolated to infinity observed from

Tlast to infinity. 